\title{
Estudo de potenciais interações medicamentosas em pacientes hipertensos
}

\author{
Hypertensive patients and potential drug-drug interactions
}

Recebido em: 21/12/2014 Aceito em: 28/04/2015

\author{
Sally Cristina Moutinho MONTEIRO', Ilka Kassandra Pereira \\ BELFORT ${ }^{2}$, Wandson Rodrigues SOUSA1, Clemilson da Silva BARROS ${ }^{1}$, \\ Karla Valéria Santos de CAMPOS ${ }^{1}$ \\ 'Programa de Pós- Graduação em Saúde do Adulto e da Criança, Universidade Federal do \\ Maranhão - UFMA. Av. dos Portugueses, 1966. Bacanga-CEP 65080-805. São Luís, MA, Brasil. \\ Universidade Aberta do SUS - UNASUS, Universidade Federal do Maranhão - UFMA. Rua Viana \\ Vaz, No 41, Centro, CEP: 65020-660,São Luis, MA, Brasil.E-mail: sallycris@yahoo.com.br
}

\begin{abstract}
Hypertension is a chronic elevation of systolic and diastolic blood pressure and is probably the most common chronic disease. These patients require special attention, since many of them use more than one drug, thereby increasing the risk of drug-drug interactions. These interactions may increase morbidity and mortality if preventive measures are not taken. The aim of this work was to investigate potential drug interactions in hypertensive patients from a unit of family health strategy in São Luís, MA. An observational quantitative cross-sectional study was carried out with 60 hypertensive patients from System of Registration and Monitoring Hypertensive Diabetics (SIS-HIPERDIA) linked to a Basic Health Unit (BHU) of São Luís, MA. The subjects were predominantly female (71.67\%), Afro-American $(46.66 \%)$, with incomplete primary education $(55 \%)$ and family income of up to half the minimum wage $(65 \%)$. It was found 70 potential drug interactions, where stood out the moderate interactions $(84.28 \%)$. The therapeutic class most frequently involved in drug-drug interactions was anti-inflammatory, and the more frequent associations occurred between acetylsalicylic acid with losartan, and hydrochlorothiazide with captopril. The results showed a high prevalence of potential drug-drug interactions in the studied population, highlighting the moderate interactions, confirming data in the literature. The search of collaborative work between doctors and pharmacists should be constant, aimed at intensifying the health care process, knowledge sharing and comprehensive care.
\end{abstract}

Keywords: pharmaceutical care, hypertension, drug-drug interactions

\section{RESUMO}

Hipertensão arterial é uma elevação crônica da pressão arterial sistólica e diastólica e é provavelmente a doença crônica mais comum nos dias de hoje. Os portadores desse agravo necessitam de atenção especial, uma vez que muitos fazem uso de associação de medicamentos, aumentando assim o risco de interações medicamentosas. O objetivo do trabalho foi verificar potenciais interações medicamentosas com anti-hipertensivos em usuários hipertensos de uma estratégia de saúde da família, em São Luís. MA. Foi realizado um estudo quantitativo observacional de corte transversal com 60 hipertensos do Sistema de Cadastramento e Acompanhamento de Hipertensos e Diabéticos (SIS-HIPERDIA), vinculado a uma Unidade Básica de Saúde (UBS) de São Luís, MA. A população estudada foi predominantemente do sexo feminino $(71,67 \%)$, de cor parda (46,66\%), com ensino fundamental incompleto $(55 \%)$ e renda familiar de até meio salário mínimo (65\%). Foram observadas 70 potenciais interações medicamentosas, onde destacaram-se as interações moderadas $(84,28 \%)$. As classes terapêuticas mais frequentemente envolvidas foram os anti-inflamatórios e as associações mais frequentes ocorreram entre ácido acetilsalicílico com losartana e hidroclorotiazida com captopril. Os resultados mostraram alta prevalência de potenciais interações medicamentosas na população estudada, ratificando dados encontrados na literatura. A busca do trabalho colaborativo entre prescritores e farmacêuticos deve ser constante objetivando a intensificação do processo de cuidados em saúde, o compartilhamento de conhecimentos e a integralidade do cuidado.

Palavras chave: atenção farmacêutica; hipertensão; interações medicamentosas 


\section{INTRODUÇÃO}

A hipertensão arterial sistêmica (HAS) tem sua origem em questões multifatoriais, sendo caracterizada clinicamente por níveis elevados e sustentados da pressão arterial (PA) ( $\geq 140$ x $90 \mathrm{mmHg}$ ), estando frequentemente associada a alterações funcionais e/ou estruturais dos órgãos-alvo como: coração, encéfalo, rins e vasos sanguíneos; e a alterações metabólicas, com aumento do risco de eventos cardiovasculares fatais e não fatais $(1,2)$.

De acordo com dados da Vigilância de Fatores de Risco e Proteção para Doenças Crônicas por Inquérito Telefônico (VIGITEL), o diagnóstico médico prévio de HAS alcançou 23,3\% em 2010, sendo ligeiramente maior em mulheres $(25,5 \%)$ do que em homens $(20,7 \%)$. Em ambos os sexos, o diagnóstico de HAS se torna mais comum com o envelhecimento, alcançando cerca de $50 \%$ na faixa etária 55 anos ou mais. Esse quantitativo vem crescendo com a ocorrência cada vez mais frequente de doenças crônicas, envelhecimento populacional, hábitos de vida não saudáveis, entre outros (3).

O plano terapêutico a ser executado com o paciente hipertenso deve ser elaborado e acompanhado de forma efetiva com o objetivo de atingir e manter uma pressão arterial abaixo de $140 \times 90 \mathrm{mmHg}$, utilizando medidas gerais de tratamento (gerenciamento do estresse, consumo moderado de sal, exercício físico regular e redução de outros fatores de risco) e utilização de medicamento anti-hipertensivo (diuréticos, alfa- e beta-bloqueadores, bloqueadores dos canais de cálcio, inibidores da enzima conversora de angiotensina, entre outros) $(2,4)$.

No que diz respeito ao tratamento medicamentoso, constantemente ocorrem associações de anti-hipertensivos, fato que permite obtenção de efeito sinérgico ou aditivo dos medicamentos, elevando assim as chances de sucesso em hipertensos que não alcançam o controle ideal da pressão com a monoterapia. Além disso, esses pacientes necessitam de outros medicamentos de uso contínuo devido entidades clínicas associadas (diabetes, dislipidemia, doença coronariana, osteoartopatia, entre outros) ou complicações do próprio quadro hipertensivo (5-8).

A polimedicação geralmente está associada ao tratamento medicamentoso para hipertensão arterial e essa situação os torna mais susceptíveis a interações medicamentosas em níveis distintos, podendo interferir na qualidade do tratamento e de vida (9-12).

Interação medicamentosa (IM) é entendida como um evento clínico onde os efeitos de um fármaco são alterados pela presença de outro fármaco, alimento, bebida ou algum agente químico ambiental (9). Potenciais interações medicamentosas podem aumentar os efeitos indesejados dos medicamentos, acarretar ineficácia terapêutica e/ou repercutir negativamente na saúde do paciente, gerando custos elevados com o tratamento e até mesmo evoluir para o óbito do indivíduo $(13,14,15)$.

Desta forma, o objetivo do trabalho foi verificar potenciais interações medicamentosas com anti-hipertensivos utilizados por usuários hipertensos cadastrados e acompanhados no Sistema de Cadastramento e Acompanhamento de Hipertensos e Diabéticos (SIS-HIPERDIA) de uma Estratégia de Saúde da Família, em São Luís/MA.

\section{MÉTODO}

Foi realizado um estudo observacional de corte transversal com pacientes hipertensos cadastrados e acompanhados no Programa do Sistema de Cadastramento e Acompanhamento de Hipertensos e Diabéticos (SIS-HIPERDIA), atendidos por uma equipe da Estratégia de Saúde da Família (ESF) vinculada a uma Unidade Básica de Saúde (UBS), em São Luís, Maranhão.

A ESF possui 257 usuários hipertensos cadastrados. Durante o período de setembro de 2013 a outubro de 2014, todos os pacientes ambulatoriais hipertensos que foram à consulta com a enfermagem foram identificados como potenciais candidatos para o estudo. Os critérios de exclusão, consultados nos prontuários, foram: estágio 3 de hipertensão (pressão sistólica de $180 \mathrm{mmHg}$ e/ou pressão diastólica de $110 \mathrm{mmHg}$ ), causa secundária identificada (diagnosticada) de hipertensão (tais como a doença renal crônica, feocromocitoma, síndrome de Cushing).

Quanto aos critérios de não inclusão: maiores de 18 anos e estar cadastrado e acompanhado no SIS-HIPERDIA. Além disso, precisava preencher três ou mais dos critérios descritos a seguir: frequentar a UBS regularmente (pelo menos uma vez por mês), possuir medicação anti-hipertensiva prescrita, ser polimedicado (utilização de 5 ou mais medicamentos concomitantemente), possuir comorbidades e história de inefetividade da terapêutica medicamentosa. O critério de exclusão foi definido como a ausência em um ou mais encontros durante o estudo. A Figura 1 mostra o fluxo da seleção dos participantes.

Os pacientes foram agendados para comparecimento a UBS para uma entrevista com questionário semiestruturado, contendo variáveis sócio demográficas, de saúde, e terapia medicamentosa. 
257 Usuários Hipertensivos Cadastrados

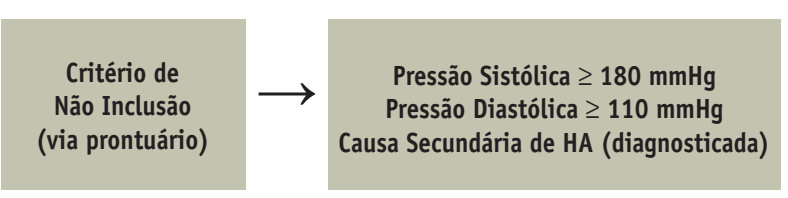

242 Usuários Hipertensivos Elegiveis

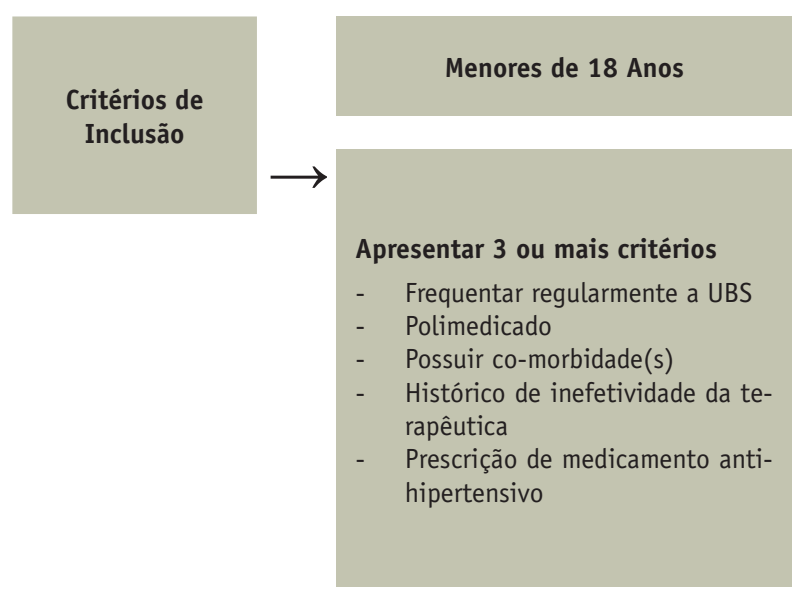

220 Usuários Hipertensos Elegiveis

75 Usuários Entrevistados e Acompanhados

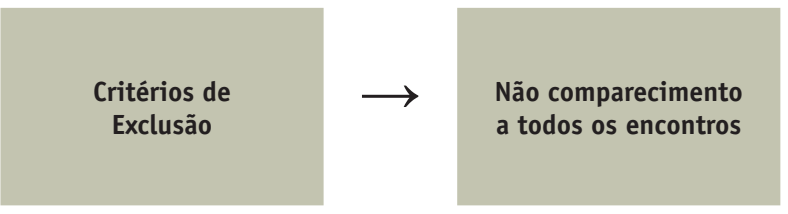

Amostra Final $=60$ Usuários Hipertensos

Figura 1 - Representação gráfica do processo de seleção dos participantes da pesquisa.

A entrevista foi com escuta ativa e passiva. Posteriormente foi realizado a análise da situação de saúde e histórico de medicações, identificação das necessidades e problemas, avaliação das interações medicamentosas quanto à relevância clínica, documentação e quando necessária intervenção (comunicado ao médico e a enfermeira). A duração da entrevista consistia em 30 a 40 minutos. Na data da entrevista foi realizada a aferição de pressão arterial utilizando esfigmomanômetro aneroide e estetoscópico (Premium ${ }^{\circledR}$ ), observando as recomenda- ções contidas nas VI Diretrizes Brasileiras de Hipertensão (2); bem como a concentração da glicemia capilar (em jejum de 8 horas) utilizando lancetas estéreis, tiras reativas e glicosímetro da linha Accu-ChekAdvange II (Roche $\left.{ }^{\circledR}\right)$, considerando as orientações da Sociedade Brasileira de Diabetes - SBD (16). Também foi realizada a verificação do peso (em quilos) e altura (em metros) para o cálculo do índice de massa corpórea (IMC). A determinação dos valores do peso $(\mathrm{Kg})$ foi realizada pela balança digital Toledo ${ }^{\circledR}$ e a medição da altura, pelo estadiômetro manual da marca Alturaexata ${ }^{\circledR}$. Foi considerado como sobrepeso o IMC maior que $25 \mathrm{~kg} / \mathrm{m}^{2}$, e como obesidade IMC maior que $30 \mathrm{~kg} / \mathrm{m}^{2}$, calculados a partir do quociente entre o peso e o quadrado da altura (17).

O projeto seguiu a Resolução da CONEP 466/12 e foi aprovado pelo Comitê de Ética em Pesquisa do Hospital Universitário da Universidade Federal do Maranhão com parecer de número 289.937.

Foram utilizadas as monografias dos fármacos da base de dados Drugdex System - Thomson Micromedex ${ }^{\circledR}$ - Interactions, a fim de verificar o registro de possíveis interações entre os medicamentos utilizados pelos usuários (18). As associações que apresentaram potencial de interação foram classificadas e apresentadas de acordo com a gravidade conforme a mesma base de dados. De acordo com o software Micromedex ${ }^{\circledR}$ as interações foram classificadas em potencialmente graves (que podem oferecer risco de morte e/ou requerer intervenção médica urgente para minimizar efeitos adversos graves), moderadas (que podem resultar em exacerbação das condições clínicas do paciente e/ou requererem troca de terapia) e leves (interações com efeitos clínicos limitados, podendo sua manifestação incluir aumento da frequência ou severidade dos efeitos adversos, mas que não requerem alterações importantes na terapia).

Os dados obtidos foram organizados soba forma de tabelas e gráficos com auxílio do software Microsoft Excel ${ }^{\circledR}$ (versão 2010) e software Microsoft Word ${ }^{\circledR}$ (versão 2010) para o processamento de texto. Foi utilizada estatística descritiva (percentual, média e desvio padrão), para um melhor entendimento dos resultados.

\section{RESULTADOS}

Participaram do estudo 60 pacientes, com média de idade $61( \pm 11,23)$ anos, dos quais $43(71,6 \%)$ pertencentes ao sexo feminino. As Tabelas 1 e 2 mostram a distribuição de frequência dos dados demográficos, socioeconômicos e de indicadores de saúde na população estudada. 
Tabela 1 - Distribuição de frequência dos indicadores demográficos e socioeconômicos de uma amostra de hipertensos cadastrados na Estratégia Saúde da Família (ESF) do município de São Luís, MA.

\begin{tabular}{|c|c|c|}
\hline & Masculino & Feminino \\
\hline Gênero & $17(28,33 \%)$ & $43(71,67 \%)$ \\
\hline Média de Idade & $58,88 \pm 11,0$ & $61,83 \pm 11,21$ \\
\hline \multicolumn{3}{|l|}{ Cor (auto declarada) } \\
\hline Pardo & $5(8,33 \%)$ & $23(38,33 \%)$ \\
\hline Preto & $6(10 \%)$ & $13(21,67 \%)$ \\
\hline Branco & $5(8,33 \%)$ & $5(6,68 \%)$ \\
\hline Indígena & $2(3,33 \%)$ & $2(3,33 \%)$ \\
\hline \multicolumn{3}{|l|}{ Escolaridade } \\
\hline Analfabeto & 0 & $7(11,67 \%)$ \\
\hline Fundamental Incompleto & $8(13,33 \%)$ & $25(41,67)$ \\
\hline Fundamental Completo & $6(10 \%)$ & $4(6,67 \%)$ \\
\hline Médio Incompleto & $1(1,67 \%)$ & $2(3,33 \%)$ \\
\hline Médio Completo & $2(3,33 \%)$ & $5(8,33 \%)$ \\
\hline \multicolumn{3}{|l|}{ Estado Civil } \\
\hline Casado (a) & $11(18,33 \%)$ & $20(35 \%)$ \\
\hline Solteiro (a) & $4(6,67)$ & $11(16,67 \%)$ \\
\hline Viúvo (a) & $2(3,33 \%)$ & $10(16,67 \%)$ \\
\hline Divorciado (a) & 0 & $2(3,33 \%)$ \\
\hline \multicolumn{3}{|l|}{ Renda Familiar Mensal } \\
\hline Sem rendimento & 0 & $3(5 \%)$ \\
\hline Até $1 / 2$ salário mínimo & $9(15 \%)$ & $27(45 \%)$ \\
\hline > 1/2 a 1 salário mínimo & $7(11,67 \%)$ & $8(13,33 \%)$ \\
\hline > 1 a 2 salários mínimos & $1(1,67 \%)$ & $5(10 \%)$ \\
\hline
\end{tabular}

Em relação às morbidades encontradas 3,33\% dos entrevistados relataram doença cardíaca, 3,33\% síndrome do pânico, 5\% doença reumática, 8,33\% osteopenia, $1,67 \%$ câncer e $56,67 \%$ diabetes mellitus.

$\mathrm{O}$ valor médio da pressão arterial sistólica (PAS) foi de $151,02( \pm 25,16) \mathrm{mm} \mathrm{Hg}$ e a diastólica (PAD) de $92,37( \pm 23,19) \mathrm{mm} \mathrm{Hg}$. Sendo que 58,3\% (28 mulheres e 7 homens) encontram-se com pressão arterial fora dos valores desejáveis $(\leq 140 / 90 \mathrm{~mm} \mathrm{Hg})$ indicando falta de efetividade do tratamento utilizado.
Tabela 2 - Distribuição de frequência de indicadores de saúde de uma amostra de hipertensos cadastrados na Estratégia Saúde da Família (ESF) do município de São Luís, MA.

\begin{tabular}{|c|c|c|}
\hline Gênero & Masculino & Feminino \\
\hline $\begin{array}{l}\text { Média de tempo } \\
\text { de Diagnóstico de } \\
\text { Hipertensão }\end{array}$ & $\pm 9,26$ & $\pm 8,65$ \\
\hline \multicolumn{3}{|c|}{ Histórico Familiar de Hipertensão } \\
\hline Sim & $14(23,33 \%)$ & $33(55 \%)$ \\
\hline Não & $2(3,34 \%)$ & $7(11,67 \%)$ \\
\hline Não sabe & $1(1,67 \%)$ & $3(5 \%)$ \\
\hline \multicolumn{3}{|c|}{ IMC } \\
\hline Eutrófico & $2(3,33 \%)$ & $12(20 \%)$ \\
\hline Sobrepeso & $9(15 \%)$ & $15(25 \%)$ \\
\hline Obesidade grau I & $6(10 \%)$ & $11(18,33 \%)$ \\
\hline Obesidade grau II & 0 & $2(3,33 \%)$ \\
\hline Obesidade grau III & 0 & $3(5 \%)$ \\
\hline \multicolumn{3}{|c|}{ Glicemia Capilar } \\
\hline$\leq 70$ & 0 & $5(8,33 \%)$ \\
\hline 70 a 99 & $8(13,33 \%)$ & $20(33,34 \%)$ \\
\hline 100 a 126 & $3(5 \%)$ & $6(10 \%)$ \\
\hline$\geq 126$ & $6(10 \%)$ & $12(20 \%)$ \\
\hline \multicolumn{3}{|c|}{ Diabético } \\
\hline Sim & $07(11,67 \%)$ & $27(45 \%)$ \\
\hline Não & $10(16,67 \%)$ & $16(26,66 \%)$ \\
\hline \multicolumn{3}{|c|}{ Atividade Fisica } \\
\hline Sim & $6(10 \%)$ & $8(13,33 \%)$ \\
\hline Não & $11(18,33 \%)$ & $35(58,33 \%)$ \\
\hline \multicolumn{3}{|c|}{ Tabagismo } \\
\hline Sim & $4(6,67 \%)$ & $3(5 \%)$ \\
\hline Não & $6(10 \%)$ & $28(46,67 \%)$ \\
\hline Ex-Fumante & $7(11,67 \%)$ & $12(20 \%)$ \\
\hline \multicolumn{3}{|c|}{ Etilista } \\
\hline Sim & $6(10 \%)$ & $35(58,33 \%)$ \\
\hline Não & $11(18,33 \%)$ & $8(13,33 \%)$ \\
\hline
\end{tabular}


Os resultados da glicemia capilar estavam em sua maioria dentro dos valores normais considerando as orientações da SBD (16), 13,33\% e 33,34\% em homens e mulheres respectivamente. Entretanto, pode-se observar que $20 \%$ das mulheres estavam com a glicemia elevada.

Com relação à utilização da medicação antihipertensiva verificou-se que $2(3,33 \%)$ pacientes relatam utilizá-la apenas quando sentem algum desconforto (dores de cabeça, dores no peito, tontura, fraqueza, visão embasada, entre outros). Dentre os antihipertensivos mais utilizados estão a hidroclorotiazida $(27,40 \%)$, o captopril $(15,47 \%)$ e a losartana potássica $(21,42 \%)$ (Figura 2).

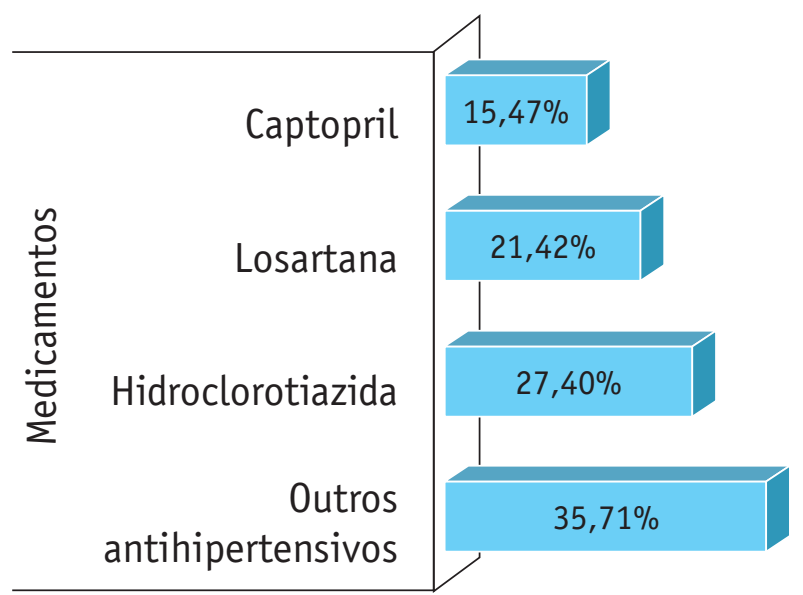

Figura 2. Distribuição percentual dos medicamentos antihipertensivos mais utilizados pelos usuários da Unidade Básica de Saúde de São Luís, MA.

No momento da entrevista, nenhum usuário relatou apresentar sintomas de hipertensão, hipoglicemia ou hiperglicemia e/ou episódios de efeitos adversos a medicamentos. Contudo, de acordo com a SBD (16) a hipertensão arterial e a diabetes mellitus são condições clínicas que quando se associam, aumentam consideravelmente o risco de morbidade e mortalidade cardiovasculares.
O consumo de medicamentos foi em média de 2,4 por indivíduo, com relato de 139 medicamentos diferentes. A presença de polifarmácia foi verificada em $8,3 \%$ dos usuários e um total 70 potenciais interações medicamentosas com anti-hipertensivos foram identificadas, sendo que $8(11,42 \%)$ foram consideradas leves, $59(84,28 \%)$ moderadas e $3(4,29 \%)$ de maior gravidade.

Potenciais interações medicamentosas moderadas (PIMM) foram as mais frequentes, ocorrendo em $30(50 \%)$ dos entrevistados. Os medicamentos mais frequentemente envolvidos em PIMM foram AAS + losartana e hidroclorotiazida + captopril. Potenciais interações medicamentosas graves (PIMG) ocorreram em $3(4,29 \%)$ de todos os entrevistados. Os medicamentos envolvidos foram enalapril + losartana. A Tabela 3 apresenta um resumo da descrição das potenciais interações medicamentosas encontradas.

\section{DISCUSSÃO}

Os participantes deste estudo apresentaram baixo grau de escolaridade, o que os torna 5 vezes mais propensos a problemas de saúde, em geral (19), devido ao baixo nível de conhecimento e compreensão, por parte deste indivíduo (20). Nesta situação a atenção farmacêutica pode contribuir de forma significativa para a compreensão e consciência quanto a sua saúde, estimulando a troca de experiência e promovendo o uso racional de medicamento. A proximidade do profissional farmacêutico junto à equipe de saúde e comunidade auxilia na implantação de projetos de promoção, prevenção e recuperação da saúde. Ressalta-se que esse profissional auxilia no processo de cuidados em saúde e na resolutividade das ações em saúde, buscando o uso racional de medicamentos, segurança em saúde e melhoria da qualidade de vida dos clientes/pacientes.

Tabela 3 - Potenciais interações medicamentosas e sua relevância clínica em usuários de Estratégia Saúde da Família (ESF) do município de São Luís, MA.

\begin{tabular}{|l|c|c|c|}
\hline \multicolumn{1}{|c|}{ Interação Potencial } & n (casos) & $\mathbf{f ~ ( \% )}$ & Relevância clínica \\
\hline enalapril + losartana & $\mathbf{3}$ & $\mathbf{4 , 2 9}$ & Grave \\
\hline ácido acetilsalićlico + omeprazol & 1 & 1,43 & Leve \\
\hline hidroclorotiazida + besilato de anlodipino & 2 & 2,86 & Leve \\
\hline indapamida + besilato anlodipino & 1 & 1,43 & Leve \\
\hline ácido acetilsalićlico + torsilax & 1 & 1,43 & Leve \\
\hline atenol + ácido acetilsalićlico & 1 & 1,43 & Leve \\
\hline
\end{tabular}




\begin{tabular}{|c|c|c|c|}
\hline Interação Potencial & n (casos) & $f(\%)$ & Relevância clínica \\
\hline propanolol + a ácido acetilsalicílico & 1 & 1,43 & Leve \\
\hline hidroclorotiazida + besilato de anlodipino & 1 & 1,43 & Leve \\
\hline propanolol + hidroclorotiazida & 1 & 1,43 & Moderada \\
\hline pantropazol + hidroclorotiazida & 1 & 1,43 & Moderada \\
\hline hidroclorotiazida + calcinan & 1 & 1,43 & Moderada \\
\hline amitriptilina + diazepam & 1 & 1,43 & Moderada \\
\hline losartana + diazepam & 1 & 1,43 & Moderada \\
\hline hidroclorotiazida + citrato de cálcio & 1 & 1,43 & Moderada \\
\hline hidroclorotiazida + gingko biloba & 1 & 1,43 & Moderada \\
\hline cálcio + maleato de estrôncio & 1 & 1,43 & Moderada \\
\hline atenolol + indapamida & 1 & 1,43 & Moderada \\
\hline atenolol + besilato de anlodipino & 1 & 1,43 & Moderada \\
\hline ácido acetilsalićlico + diclofenaco de potássio & 1 & 1,43 & Moderada \\
\hline hidroclorotiazida + diclofenaco de potássio & 1 & 1,43 & Moderada \\
\hline ácido acetilsalicílico + losartana & 6 & 8,57 & Moderada \\
\hline diclofenaco de potássio + losartana & 1 & 1,43 & Moderada \\
\hline enalapril + hidroclorotiazida & 4 & 5,71 & Moderada \\
\hline captopril + ácido acetilsalicílico & 3 & 4,28 & Moderada \\
\hline captopril + hidroclorotiazida & 5 & 7,14 & Moderada \\
\hline hidroclorotiazida + amitriptilina & 1 & 1,43 & Moderada \\
\hline losartana + amitriptilina & 2 & 2,86 & Moderada \\
\hline ácido acetilsalicílico + enalapril & 2 & 2,86 & Moderada \\
\hline indapamida + benazepil & 1 & 1,43 & Moderada \\
\hline propanolol + diclofenaco de potássio & 1 & 1,43 & Moderada \\
\hline propanolol + butazona & 1 & 1,43 & Moderada \\
\hline propanolol + dorflex & 1 & 1,43 & Moderada \\
\hline cilostazol + ácido acetilsalicílico & 1 & 1,43 & Moderada \\
\hline besilato de anlodipino + torsilax & 1 & 1,43 & Moderada \\
\hline atenolol + torsilax & 1 & 1,43 & Moderada \\
\hline mesilato de doxazosina + besilato de anlodipino & 1 & 1,43 & Moderada \\
\hline ácido acetilsalicílico + besilato de anlodipino & 1 & 1,43 & Moderada \\
\hline mesilato de doxazosina + atenol & 1 & 1,43 & Moderada \\
\hline ácido acetilsalicílico + besilato anlodipino & 1 & 1,43 & Moderada \\
\hline atenol + besilato anlodipino & 1 & 1,43 & Moderada \\
\hline losartana + ácido acetilsalicílico & 1 & 1,43 & Moderada \\
\hline propanolol + natrix & 1 & 1,43 & Moderada \\
\hline metildopa + sulfato ferroso & 1 & 1,43 & Moderada \\
\hline hidroclorotiazida + alenia & 1 & 1,43 & Moderada \\
\hline ácido acetilsalicílico + besilato de anlodipino & 1 & 1,43 & Moderada \\
\hline (valsartana + hidroclorotiazida + ácido acetilsalicílico & 1 & 1,43 & Moderada \\
\hline hidroclorotiazida + besilato de anlodipino & 1 & 1,43 & Moderada \\
\hline ácido acetilsalicílico + valsartana & 1 & 1,43 & Moderada \\
\hline captopril + diclofenaco de potássio & 1 & 1,43 & Moderada \\
\hline hidroclorotiazida + diclofenaco de potássio & 1 & 1,43 & Moderada \\
\hline losartana + diclofenaco & 1 & 1,43 & Moderada \\
\hline
\end{tabular}


Neste contexto, avaliar a situação sócio-demográfica pode auxiliar a entender influências externas no cuidado em saúde desta população. Vale ressaltar, que o bairro onde este estudo foi realizado (Coroadinho, São Luís/MA), segundo os dados do censo do IBGE, é a quarta maior favela do Brasil (21), apresentando-se como área de morro, inúmeras casas em situações de risco e/ou alerta, saneamento básico precário, baixa renda familiar e baixo grau de escolaridade. Além disso, é uma área fortemente caracterizada por violência e uso de drogas ilícitas.

Foi observado que houve prevalência de usuários do sexo feminino em comparação ao sexo masculino, o que pode ser justificado pelo comportamento feminino de maior autocuidado e preocupação com a saúde. Razões semelhantes são encontradas também nos trabalhos de Santos et al. (2012) no qual o sexo feminino foi mais forte em sua participação, quando comparado ao masculino, e foi encontrada a maior motivação pela busca dos serviços em saúde por parte das mulheres (15). Silva et al. (2008) justificaram a grande participação feminina por fatores hormonais e alterações metabólicas que muitas vezes estão relacionadas ao surgimento de problemas cardiovasculares (22).

No presente estudo, os anti-hipertensivos mais utilizados foram hidroclorotiazida, losartana e captopril, como encontrado por outros autores (23-26). Um elevado número de participantes apresentou controle da sua pressão arterial inadequado, indicando assim necessidade de ajustes terapêuticos, seja por consequência da interação medicamentosa, por doses inadequadas ou por não adesão ao tratamento.

A literatura enfatiza que o profissional farmacêutico desempenha um papel importante no controle da pressão arterial, influenciando positivamente na adesão ao tratamento e uso correto do medicamento, uma vez que reafirma as orientações quanto ao uso suscitado pelos prescritores e avalia os aspectos farmacêuticos e farmacológicos que possam representar um dano em potencial a saúde do indivíduo $(27,28)$.

Dentre as 70 potenciais interações medicamentosas com anti-hipertensivos encontradas neste estudo, $50 \%$ foram PIMM, que requerem o monitoramento da terapia farmacológica, o que se assemelha ao estudo de Leão et al. (2013) (29). E apesar do tratamento anti-hipertensivo comumente utilizar múltiplos fármacos (pois esta estratégia terapêutica permite obter efeito sinérgico e/ou aditivo dos medicamentos) essa prática eleva as chances de interações medicamentosas, pode resultar em Problemas Relacionados a Medicamentos (PRM) e ser causa de Resultados Negativos ao Medicamento (RNM).
Os medicamentos mais frequentemente envolvidos em PIMM foram AAS + Losartana e Hidroclorotiazida + Captopril. Os anti-inflamatórios não esteroidais (AINE) podem atenuar os efeitos anti-hipertensivos por inibição da síntese de prostaglandinas (PG) renais e retenção de líquidos $(18,30)$. Porém, acredita-se que efeito benéfico relacionado a prevenção de doenças cardiovasculares, supere a possível elevação de pressão arterial. Segundo Codagnone Neto et al. (2010), o AAS, usado em baixas doses ( 80 a $200 \mathrm{mg}$ ), para prevenção de acidentes cardiovasculares, está relacionado ao aumento dos níveis pressóricos quando em uso crônico, sobretudo no paciente idoso (31). Neste contexto essa associação deve ser acompanhada para que haja devida monitoração da pressão arterial, da função renal e riscos de hemorragia gastrointestinal $(18,32,33)$.

Indivíduos que fazem uso de diuréticos (principalmente aqueles que iniciaram a terapia recente) podem apresentar súbita queda da pressão arterial na primeira hora após a ingestão da dose inicial de captopril, o que é uma resposta hipotensiva transitória e não uma contraindicação estabelecida. A associação de captopril à hidroclorotiazida, em dose baixa, oferece vantagens características do anti-hipertensivo ideal, tais como melhor controle da hipertensão arterial, redução da mortalidade cardiovascular, proteção cardíaca e renal e baixa incidência de efeitos colaterais. Assim, o benefício da associação, supera o risco de hipotensão postural por vasodilatação e depleção do volume intravascular $(32,33)$. Mas deve ser enfatizada a necessidade de avaliar cada caso de forma individualizada e contextualizada.

Potenciais interações medicamentosas graves (PIMG) ocorreram em 3 (4,29\%) de todos os entrevistados, onde foi observada a associação entre Enalapril + Losartana. Essa associação pode elevar os riscos de hipotensão, disfunção renal e hipercalemia (elevação de potássio sanguíneo), devido aos efeitos sinérgicos ou aditivos sobre o sistema renina angiotensina. A hipercalemia, em casos graves, pode levar à insuficiência renal, paralisia muscular, ritmo cardíaco irregular, e parada cardíaca. Entre o grupo de indivíduos mais propensos a desenvolver hipercalemia estão os idosos, os diabéticos, os desidratados, os cardiopatas, os diabéticos nefropatas. No entanto, se essa combinação for considerada clinicamente necessária, os eletrólitos séricos, a pressão arterial e a função renal devem ser cuidadosamente monitorados $(15,18)$.

O conhecimento das interações medicamentosas é fundamental para evitar potencial risco a saúde do paciente. Os farmacêuticos devem estar atentos para os fármacos prescritos e a medicação utilizada pelos 
pacientes, sem prescrição formal. Segundo Locatelli (2007), uma potencial interação medicamentosa pode ser prevista a partir dos conhecimentos das propriedades farmacológicas dos fármacos envolvidos, não necessariamente ocorrendo em todos os usuários (34). Deve-se, portanto, avaliar se uma interação medicamentosa está causando alterações em parâmetros clínicos e/ou laboratoriais de determinado paciente para determinar a sua relevância clínica caso a caso.

Aproximadamente $10 \%$ das interações resultam em eventos clínicos significativos, sendo a morbidade de baixo nível frequentemente observada no idoso. Diante disso e considerando a detecção de interações medicamentosas indesejáveis classificadas como sendo de alta gravidade, ressalta-se o papel do farmacêutico em informar a probabilidade de ocorrência desta interação e as principais manifestações clínicas que a mesma pode apresentar instruindo o indivíduo a relatar qualquer evento adverso a medicação utilizada (35).

Este estudo teve algumas limitações como: o número de participantes (amostra de conveniência), população restrita (população de uma equipe de estratégia de saúde da família) não sendo assim possível a generalização dos resultados e impossibilidade (pelo tipo de desenho do estudo - transversal) de verificar as possíveis repercussões clínicas das potenciais interações medicamentosas nos pacientes entrevistados.

Por outro lado, destaca o elevado número de potenciais interações medicamentosas com anti-hipertensivos, sendo na sua maioria envolvendo anti-inflamatórios, os quais podem apresentar toxicidade renal, gastrointestinal e cardiovascular. Além disso, ressalta-se o número de pacientes sem controle adequado da pressão arterial e a presença de polimedicação em uma população ido- sa. Esses fatos podem comprometer a segurança desses indivíduos, o que evidencia a relevância do tema, a necessidade de avaliar e monitorar a terapêutica medicamentosa e a importância da presença do farmacêutico para o efetivo acompanhamento farmacoterapêutico e contribuição para resolutividade das ações em saúde.

\section{CONCLUSÃO}

Os resultados mostraram uma alta prevalência de potenciais interações medicamentosas na população estudada, destacando-se as interações moderadas, ratificando dados encontrados na literatura. A frequência de interações medicamentosas é um risco permanente, principalmente entre idosos e polimedicados. Nesta perspectiva, profissionais prescritores e farmacêuticos devem averiguar constantemente a medicação utilizada pelos pacientes para identificar as possíveis interações medicamentosas e assim contribuir para a segurança do paciente e o uso racional de medicamentos. A busca do trabalho colaborativo entre prescritores e farmacêuticos deve ser constante objetivando a intensificação do processo de cuidados em saúde, o compartilhamento de conhecimentos e a integralidade do cuidado.

\section{AGRADECIMENTOS}

Aos voluntários da pesquisa e à equipe da Estratégia de Saúde da Família da Unidade Básica de Saúde Dr. Antônio Guanaré. Ao Programa de Pesquisa para o SUS (PPSUS) e à Fundação de Amparo a Pesquisa e ao Desenvolvimento Científico e Tecnológico do Maranhão (FAPEMA) pelo recurso financeiro.

\section{REFERÊNCIAS}

1. BRASIL. Ministério da Saúde. Secretaria de Atenção à Saúde. Departamento de Atenção Básica. Hipertensão Arterial Sistêmica para o Sistêmico único de Saúde. Brasília: (DF); 2006.

2. SBC. Sociedade Brasileira de Cardiologia. VI Diretrizes Brasileiras de Hipertensão. Arq. Bras. Cardiol., São Paulo, 2010:1; 95, (1), (Suppl) 1-51.

3. Lima CLP, Rios PSS. Interações medicamentosas na hipertensão: papel do Farmacêutico no Acompanhamento Clínico dos Pacientes. Cadernos de Graduação - Ciências Biológicas e da Saúde -, Aracajú, 1998. 13 (14):69-81.
4. BRASIL. Ministério da Saúde. Secretaria de Atenção à Saúde. Departamento de Atenção Básica. Estratégia para o cuidado da pessoa com doença crônica hipertensão arterial sistêmica. Brasília: (DF); 2013.

5. Moreno AH, Nogueira EP, Perez MPMS, Lima LRO. Atenção farmacêutica na prevenção de interações medicamentosas em hipertensos. Rev Inst Ciênc Saúde, 2007. 4 (25):373- 7 .

6. Silva MEDC. Representações sociais da hipertensão arterial elaboradas por portadas e profissionais de Saúde: uma contribuição para a enfermagem [Monografia]. Teresina: Universidade Federal do Piauí; 2010. 
